\title{
NOTE ON CLASSES OF FUNCTIONS DEFINED BY INTEGRATED LIPSCHITZ CONDITIONS ${ }^{1}$
}

\author{
BY J. L. WALSH
}

Communicated by Maurice Heins, November 1, 1967

The classes of functions with period $2 \pi$ defined in terms of specific integrated Lipschitz conditions were characterized also in terms of degree of mean trigonometric approximation by Hardy and Littlewood without proof, results proved later by Quade [1], and still later supplemented by Zygmund [2] in his study of smooth functions. Analogs on approximation by complex polynomials of these results are due to Walsh and Russell [3]. The relative inclusion properties of these classes are difficult to treat directly, but certain results can be readily obtained by means of polynomial approximation properties, as is the purpose of this note to indicate.

A function $f(w)$ analytic in $|w|<1$ is said (Hardy) to be of class $H_{p}(p>1)$ there if the $p$ th power norms of $f\left(r e^{i \phi}\right)$ with $r$ fixed are uniformly bounded for $0<r<1$; under these conditions, boundary values $f\left(e^{i \phi}\right)$ for approach "in angle" as $r \rightarrow 1$ exist for almost all $\phi$, and $\int_{0}^{2 \pi}\left|f\left(e^{i \phi}\right)\right|^{p} d \phi$ exists. A function $f(z)$ analytic in the interior of an analytic Jordan curve $C$ in the $z$-plane is of class $H_{p}(p>1)$ there if its transform is of class $H_{p}$ when the interior of $C$ is mapped onto $|w|<1$. Such a function is of class $H_{p}(k, \alpha)$ on $C, p>1,0<\alpha<1$, provided $f^{(k)}(z)$ exists on $C$ and

$$
\int c\left|f_{1}^{(k)}(s+h)-f_{1}^{(k)}(s)\right|^{p}|d z| \leqq A|h|^{p \alpha}
$$

where $s$ indicates arc length on $C$ and $f_{1}{ }^{(k)}(s)$ is the $k$ th derivative of $f(z)$ with respect to $s$ on $C$. Here and below $A$ represents a constant independent of $s$ and $z$ which may change from one inequality to another. The Zygmund condition for $\alpha=1$ replaces (1) by

$$
\int_{C}\left|f_{1}^{(k)}(s+h)+f_{1}^{(k)}(s-h)-2 f_{1}^{(k)}(s)\right|^{p}|d z| \leqq A|h|^{p}, \quad p>1,
$$

and defines functions (of class $H_{p}$ ) of class $H_{p}(k, 1)$.

An analog [3] of the results on trigonometric approximation already mentioned is

${ }^{1}$ Research supported in part by the Air Force Office of Scientific Research, Air Research and Development Command. Abstract published under another title in Notices of the American Mathematical Society, Vol. 14 (1967), pp. 526. 
THEOREM 1. If $C$ is an analytic Jordan curve in the z-plane, then a necessary and sufficient condition that $f(z)$ be of class $H_{p}(k, \alpha)$ on $C$ is that there exist polynomials $p_{n}(z)$ of respective degrees $n$ in $z$ such that $(p>1)$

(3) $\int_{C}\left|f(z)-p_{n}(z)\right| p|d z| \leqq A_{1} / n^{p(k+\alpha)}, \quad 0<\alpha \leqq 1, \quad k \geqq 0$.

We have shown elsewhere [4], by study of the polynomial series involved, that (3) implies $(1<p<q \leqq \infty)$

$$
\int_{C}\left|f(z)-p_{n}(z)\right| q|d z| \leqq A_{2} / n^{q(k+\alpha)-q / p+1},
$$

which by Theorem 1 implies that $f(z)$ is of class $H_{q}([\beta], \beta-[\beta])$ on $C$, where $[\beta]$ denotes the largest integer less than $\beta$, where $\beta=k+\alpha$ $-1 / p+1 / q$, and we suppose $\beta>0$. Consequently we have our main result:

THEOREM 2. If $f(z)$ is of class $H_{p}(k, \alpha)$ on the analytic Jordan curve $C, p>1, k \geqq 0,0<\alpha \leqq 1$, then $f(z)$ is also of class $H_{q}([\beta], \beta-[\beta])$ on $C$ provided $\beta=k+\alpha-1 / p+1 / q>0$ and $1<p<q \leqq \infty$.

Of course, when a function is defined by an integral it may be defined arbitrarily on a set of measure zero; here $f^{(k)}(z)$ [or $f^{(k-1)}(z)$ ] may be defined as continuous on $C$ if $\alpha>1 / p$ [or $k>0$ ].

Theorems 1 and 2 can be modified [4] so as to remain valid for a function $f(z)$ defined merely on $C$. We say that a function $f(z)$ defined on $C$ and satisfying (1), or (2), is of class $L_{p}(k, \alpha)$ according as $0<\alpha<1$ or $\alpha=1$. We replace the polynomials in (3) and (4) by polynomials $p_{n}(z, 1 / z)$ of respective degrees $n$ in $z$ and $1 / z$, where the origin $O$ lies interior to $C$. Theorem 1 as modified asserts that (3) as modified is necessary and sufficient that $f(z)$ belong to $L_{p}(k, \alpha)$ on $C$. Theorem 2 as modified asserts that if $f(z)$ is of class $L_{p}(k, \alpha)$ on $C, p>1, k \geqq 0$, $0<\alpha \leqq 1$, then $f(z)$ is also of class $L_{q}([\beta], \beta-[\beta])$ on $C$ provided $\beta=k+\alpha-1 / p+1 / q>0$ and $1<p<q \leqq \infty$. In particular, if $C$ is the unit circumference $|z|=1$, a polynomial $p_{n}(z, 1 / z)$ of degree $n$ in $z$ and $1 / z$ is also a trigonometric polynomial in $\phi=\arg z$ of order $n$, and conversely. So the hypothesis of modified Theorem 2 refers on $C$ only to classes $L_{p}(k, \alpha)$; it shows that if $f\left(e^{i \phi}\right)$ (necessarily with period $2 \pi$ ) is of class $L_{p}(k, \alpha)$ on $C:|z|=1$ with respect to $\phi$, then $f\left(e^{i \phi}\right)$ is also of class $L_{q}([\beta], \beta-[\beta])$ on $C$ with respect to $\phi$, where $\beta=k+\alpha-1 / p$ $+1 / q>0,1<p<q \leqq \infty$. 


\section{REFERENCES}

1. E. S. Quade, Trigonometric approximation in the mean, Duke Math. J. 3 (1937), 529-543.

2. A. Zygmund, Smooth functions, Duke Math. J. 12 (1945), 47-76.

3. J. L. Walsh and H. G. Russell, Integrated continuity conditions and degree of approximation by polynomials or by bounded analytic functions, Trans. Amer. Math. Soc. 92 (1959), 355-370.

4. J. L. Walsh, Approximation by polynomials: uniform convergence as implied by mean convergence, Proc. Nat. Acad. Sci. U.S.A. 55 (1966), 20-25; 1405-1407; 56 (1966), 1406-1408.

UNIVERSITY OF MARYLAND 